\title{
Glyceryl trinitrate blocks staphyloxanthin and biofilm formation in Staphylococcus aureus
}

\author{
Hisham A Abbas ${ }^{1}$, Ahmed M Elsherbini' ${ }^{2}$, Moutaz A Shaldam ${ }^{3}$
}

1. Department of Microbiology and Immunology-Faculty of Pharmacy-Zagazig University- Zagazig- Egypt.

2. Health Sciences College-Umm Al Qura University, AlQunfudah, Saudi Arabia.

3. Department of Medicinal Chemistry, Faculty of Pharmacy, Delta University for Science and Technology, Gamasa, Egypt.

\begin{abstract}
Background: Staphylococcus aureus is an important nosocomial bacterium that is responsible for a number of infections that may be fatal. The treatment of such infections is limited by emergence of antibiotic resistance. Targeting virulence of Staphylococcus aureus may provide an alternative option to antibiotic that may bypass the emergence of resistant strains due to lack of stress on viability.

Objectives: Investigation of the ability of glyceryl trinitrate (GTN) to inhibit staphyloxanthin, biofilm and tolerance to oxidative stress.

Methods: The disk sensitivity method was used to detect the methicillin resistance of Staphylococcus aureus. The effect of sub-inhibitory concentration of GTN on biofilm formation, staphyloxanthin production and tolerance to oxidative stress was evaluated. Molecular docking study was used to investigate the ability of GTN to bind to dehydrosqualene synthase enzyme.

Results: GTN showed a significant inhibition of biofilm, staphyloxanthin and tolerance to oxidative stress. In the molecular docking study, it was found that GTN could bind to dehydrosqualene synthase enzyme by hydrogen bonding,electrostatic interaction and pi-cation interaction.

Conclusion: The present study revealed the ability of GTN to serve as a potential anti-virulence candidate for attenuation of S. aureus pathogenicity.

Keywords: Glyceryl trinitrate, Staphylococcus aureus, staphyloxanthin, biofilm.

DOI: https://dx.doi.org/10.4314/ahs.v19i1.10

Cite as: Abbas HA, Elsherbini AM, MA S. Glyceryl trinitrate blocks staphyloxanthin and biofilm formation in Staphylococcus aureus. Afri Health Sci. 2019;19(1). 1376-1384. bttps:/ /dx.doi.org/10.4314/abs.v19i1.10
\end{abstract}

\section{Introduction}

Unlike the large number of antibiotics discovered or synthesized in the twentieth century,the number of antibiotics developed in the current century is much lower ${ }^{1}$. Moreover, antibiotics prescribed for treatment of infections over many years led to the emergence of antibiotic

\section{Corresponding author:}

Hisham Abdel Monem Abdel Hamid Abbas,

Address: Egypt, Zagazig, Zagazig University,

Faculty of Pharmacy,

Department of Microbiology and Immunology.

Fax No.:(002)0552303266

Telephone No: (002)01021878670

Email: hishamabbas2008@gmail.com resistant strains of Staphylococcus aureus due to the stress exerted on bacterial growth ${ }^{2,3}$. One approach of combating antibiotic resistance is based on disarming the bacterial virulence factors that enable the bacteria to cause disease. This strategy avoids the bacterial cell viability-stress induced emergence of resistance and, as a result, can be helpful in preventing or treating infections $\mathrm{s}^{3,4}$.

S. aureus is a human pathogen that is responsible for several nosocomial and community-acquired infections such as cutaneous and soft-tissue infections in addition to dangerous systemic infection ${ }^{5-7}$. Methicillin-resistant $S$. aureus (MRSA) is a great public-health problem ${ }^{8-10}$. MRSA are resistant to the majority of available antibiotics and the treatment options are very few ${ }^{11}$.

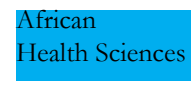

(C) 2019 Abbas. Licensee African Health Sciences. This is an Open Access article distributed under the terms of the Creative commons Attribution License (https://creativecommons.org/licenses/BY/4.0), which permits unrestricted use, distribution, and reproduction in any medium, provided the original work is properly cited. 
In order to develop multidrug resistance, it employs an arsenal of virulence factors that help it evade the host immune response. Staphyloxanthin; the golden yellow carotenoid pigment is one of such virulence factors. It acts as a reducing agent to neutralize the reactive oxygen species (ROS) produced by neutrophils and macrophages ${ }^{12}$, ${ }^{13}$. Without staphyloxanthin, S. aureus is defective in infectivity and is liable to be attacked by neutrophils with the subsequent inability to cause infection in a mouse mod$\mathrm{el}^{1,5,14}$.

Staphylococcus aureus has the ability to form biofilms either on human tissues or implants, resulting in chronic infections that are difficult to treat due to extreme resistance to antibiotics ${ }^{15}$. Inhibition of staphyloxanthin was previously investigated $^{16}$.

Glyceryl trinitrate (GTN) is a drug used in treatment of hypertension ${ }^{17}$. GTN was able to inhibit biofilms formed by Staphylococcus aureus, Staphylococcus epidermidis, Pseudomonas aeruginosa and candida albicans ${ }^{18}$. GTN is approved by FDA for topical treatment of anal fissures at concentrations up to $0.4 \%{ }^{19}$.

The objective of this study was to identify the potential inhibitory activity of glyceryl trinitrate against staphyloxanthin and biofilm formation.

\section{Materials and methods \\ Media and chemicals}

The media used in this study were Mueller Hinton broth, Mueller Hinton agar, Tryptone soya broth and Tryptone soya agar. These media in addition to methicillin antibiotic disk were purchased from Oxoid (Hampshire, UK). Glyceryl trinitrate was obtained from POHL-Boskamp, Gmbh \&Co., Hohenlockstedt, Germany (Stock solution of $1 \mathrm{mg} / \mathrm{ml})$. Other chemicals were of pharmaceutical grade.

\section{Bacterial strains}

Staphylococcus aureus ATCC 6538 strain was kindly provided by the Department of Microbiology, Faculty of Pharmacy, Mansoura University. A clinical isolate of Staphylococcus aureus (SA1) was isolated from a patient with a surgical site infection at Zagazig University Surgery Department and identified by The MALDI-TOFF apparatus at the
Clinical Pathology Department, Faculty of Medicine, Zagazig University.

\section{Antimicrobial susceptibility testing}

Antibiotic susceptibility of SA1 was tested against methicillin using the Kirby-Bauer standard disk diffusion method according to CLSI guidelines ${ }^{20}$.

Müeller-Hinton broth $(5 \mathrm{ml})$ was inoculated with three to five well-isolated colonies from an overnight agar plate culture and the broth culture was incubated at $37^{\circ} \mathrm{C}$ with shaking for 4 to 6 hours until a turbidity of a $0.5 \mathrm{McF}$ arland standard was achieved or exceeded. Sterile broth was used to adjust the turbidity to achieve turbidity matching that of a $0.5 \mathrm{McF}$ arland standard. A sterile cotton swab was moistened with the bacterial suspension and pressed on the inside wall of the tube to remove excess inoculum. The swab was streaked both over the surface of the Mueller Hinton agar plate and around the agar rim. The antibiotic disks were put on the inoculated plate and gently pressed into the agar and the plates were incubated at $37^{\circ} \mathrm{C}$ for 18 hours. The inhibition zones diameters were measured, and interpreted as resistant, intermediate or susceptible according to CLSI ${ }^{21}$.

\section{Determination of Minimum Inhibitory Concentra- tion (MIC)}

The agar dilution method was used in determination of the minimum inhibitory concentration of GTN according to the Clinical Laboratory and Standards Institute Guidelines (CLSI) ${ }^{20}$. The tested strain was incubated overnight in tryptone soya broth (TSB) and the suspension was diluted with Mueller-Hinton broth in order to prepare a suspension with a turbidity approximating that of $0.5 \mathrm{McF}$ arland Standard. The suspension was further diluted with sterile saline (1:10). By using a micropipette, a standardized inoculum (approximately $10^{4} \mathrm{CFU}$ per spot) was spotted on the surface of Mueller-Hinton agar plates containing different GTN concentrations and control plate without GTN. The MIC of GTN was the lowest concentration that inhibits growth on the plate after incubation at $37^{\circ} \mathrm{C}$ for 20 hours.

\section{Assay of biofilm formation and inhibition}

In order to test the biofilm formation capacity of $S$. aureus, the modified method of Stepanovic et al. ${ }^{22}$ was used. 
From overnight cultures of the tested strain, bacterial suspension with a density of $1 \times 10^{6} \mathrm{CFU} / \mathrm{ml}$ was prepared by dilution with TSB. The bacterial suspension was delivered in aliquots $(100 \mu \mathrm{l})$ to a sterile microtiter plate wells that was incubated for 48 hours at $37^{\circ} \mathrm{C}$. The non-adherent cells were discarded by removal of the suspensions from the microtiter plate wells and the wells were then washed 3 times using sterile phosphate buffered saline. The biofilm cells were fixed by $99 \%$ methanol for $20 \mathrm{~min}-$ utes and the wells were stained with crystal violet (1\%) for another 20 minutes followed by washing with distilled water for removing excess dye.

After leaving the plate to air dry, the dye was dissolved by $33 \%$ glacial acetic acid and the contents of the microtiter plate were transported to another plate for measuring the optical densities at $590 \mathrm{~nm}$ using spectrofluorimeter (Biotek, USA).

The cut-off optical density (ODc) was determined as three times standard deviations above the mean OD of the negative control. Following the criteria of Stepanovic et al. ${ }^{22}, S$. aureus strain was non-biofilm producer (OD less than ODc), weak biofilm producer (OD between ODc and 2x ODc), moderate biofilm producer (OD between $2 \mathrm{x}$ ODc and 4x ODc), or strong biofilm producer (OD more than $4 \mathrm{x}$ ODc).

In order to investigate the biofilm inhibiting activity of GTN, the previous procedure was repeated, but in the absence and presence of $0.125 \mathrm{mg} / \mathrm{ml}$ of GTN and biofilm inhibition was calculated by the following formula $\%$ of biofilm inhibition $=(\mathrm{OD}$ without GTN-OD with GTN)/ OD without GTN

\section{Microscopic visualization of biofilm inhibition}

$S$. aureus was grown in TSB and incubated overnight at $37^{\circ} \mathrm{C}$ and the optical density was adjusted to reach 1 at $600 \mathrm{~nm}$. Sterile glass cover slips were placed inside in 50 $\mathrm{ml}$ falcon tubes containing fresh media and inoculated with the bacterial suspension in the presence and absence of $0.125 \mathrm{mg} / \mathrm{ml}$ of GTN. The biofilms were allowed to form for 48 hours at $37^{\circ} \mathrm{C}$ and the cover slips were washed and stained with crystal violet $(1 \%)$ and examined under the light microscope using the high power (400X magnification) ${ }^{22}$.

\section{Staphyloxanthin assay}

The ability of GTN to reduce the production of the golden yellow staphyloxanthin pigment was investigated1. For qualitative assay, overnight culture of the tested strains in MHB was prepared. The bacterial suspensions were diluted 1:100 in fresh MHB containing GTN and control tubes without GTN were prepared in the same way. The tubes were incubated for 24 hours at $37^{\circ} \mathrm{C}$ with shaking at $250 \mathrm{rpm}$. The pellets were collected by centrifugation at $16,600 \times \mathrm{g}$ for $10 \mathrm{~min}$ and the pellets were photographed and the golden yellow pigment was compared in treated and untreated samples.

For quantitative assay, the pellets recovered were resuspended in methanol $(0.2 \mathrm{ml})$ by vortexing, and the resultant mixture was heated at $55^{\circ} \mathrm{C}$ for 30 minutes for pigment extraction. The extracted pigment was separated by centrifugation at $16,600 \mathrm{xg}$ for 10 minutes and the extraction process was repeated thrice. The absorbance of the extracted pigment solutions was measured at $465 \mathrm{~nm}$ using spectrofluorimeter (Biotek, USA).

\section{Sensitivity to oxidative stress}

To investigate the interfering capacity of GTN with resistance to oxidative stress, the modified disk assay method of Hassett et al. ${ }^{23}$ was used. S. aureus was cultured in TSB and incubated overnight. Aliquots of $0.1 \mathrm{ml}$ were spread onto the surface of Tryptone soya agar plates with and without $0.125 \mathrm{mg} / \mathrm{ml}$ of GTN. Sterile paper disks (6 $\mathrm{mm}$ ) were placed on the surface of Tryptone soya agar plates and $10 \mu \mathrm{l}$ of hydrogen peroxide $(1.5 \%)$ was added to them. The inhibition zones were measured after incubating the plates for 24 hours at $37^{\circ} \mathrm{C}$.

\section{Docking study}

The crystal structure of the C30 carotenoid dehydrosqualene synthase from Staphylococcus aureus was retrieved from Protein Data Bank (PDB ID: 2ZCQ) ${ }^{24}$. Docking study was carried out on glyceryl trinitrate (GTN) into the receptor active site using Molegro Virtual Docker (MVD Version 6.0). GTN was drawn into Marvin Sketch $\mathrm{V} 5.11 .5^{25}$. The most energetically favored conformer was exported as $\mathrm{mol}^{2}$ file format for docking. The optimal geometry of the ligand was determined during the docking process. The search area was set to be $13 \AA$ from the center of the active site. MolDock optimizer algorithm was chosen to perform docking process with 10 runs per ligand, 150 population size, 4000 max iteration and 8 poses for each ligand. MolDock score ${ }^{26}$ was used as scoring function. Finally, the top returned poses were chosen for analysis. 


\section{Statistical analysis}

One Way ANOVA followed by Newman-Keuls Multiple Comparison Test, Graph Pad Prism ${ }^{5}$ was used to investigate the significance of the inhibitory activities of GTN against staphyloxanthin, oxidative stress and biofilm formation. $\mathrm{P}$ values $<0.05$ were considered statistically significant.

\section{Results}

Methicillin resistance of SA1and antibacterial activity of GTN against $S$. aureus

The clinical isolate of $S$. aureus SA1 was found to be resistant to methicillin. GTN inhibited the growth of $S$. aureus at $0.5 \mathrm{mg} / \mathrm{ml}$. The inhibitory activity of GTN against biofilm formation, staphyloxanthin production and resis- tance to oxidative stress was evaluated at $1 / 4 \mathrm{MIC}(0.125$ $\mathrm{mg} / \mathrm{ml})$.

\section{GTN inhibits biofilm formation}

The standard strain $S$. aureus ATCC 6538 is well-known as a strong biofilm forming strain. The clinical isolate was also strong biofilm producer as its OD is $0.806>0.256$ (4X ODc). GTN exerted a significant biofilm inhibition in both strains. Biofilm of $S$. aureus ATCC 6538 was inhibited by $69.16 \%$, while that of the clinical isolate was reduced by $52.64 \%$ (Fig. 1). This biofilm inhibiting activity was further confirmed by microscopical examination of biofilms formed on glass cover slips in the presence and absence of GTN and examined with the light microscope. Much fewer scattered cells were found in the sample treated with GTN.

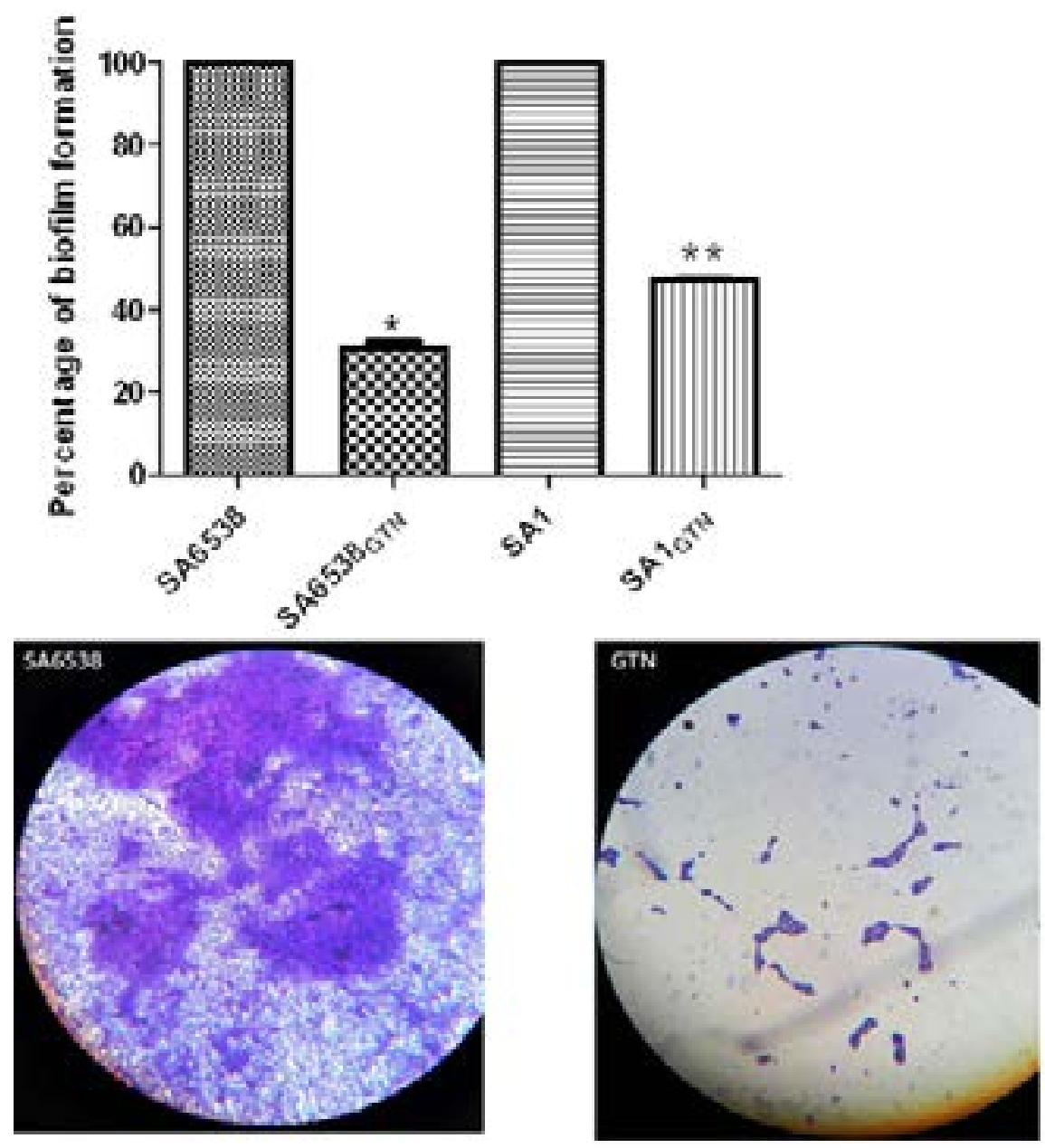

Figure 1. Inhibition of biofilm formation of S. aueus by $1 / 4$ MIC of GTN. *, significant $P<0.05$. Microscopic images of biofilm formed by $S$. aureus ATCC 6538 under the light microscope (X400) in the treated culture (right) and untreated (left). 


\section{GTN inhibits staphyloxanthin production}

The golden yellow color of staphyloxanthin enables the visual inspection of its production. The cell pellets separated from the GTN treated samples showed a remarkable reduction in staphyloxanthin production (Fig. 2).
This was confirmed by quantitative assay in which a significant inhibitory activity of staphyloxanthin was found in the GTN treated samples as compared to the control samples. The staphyloxanthinproduction was reduced by $63.37 \%$ in $S$. aureus ATCC 6538 and by $70.98 \%$ in the clinical isolate SA1.
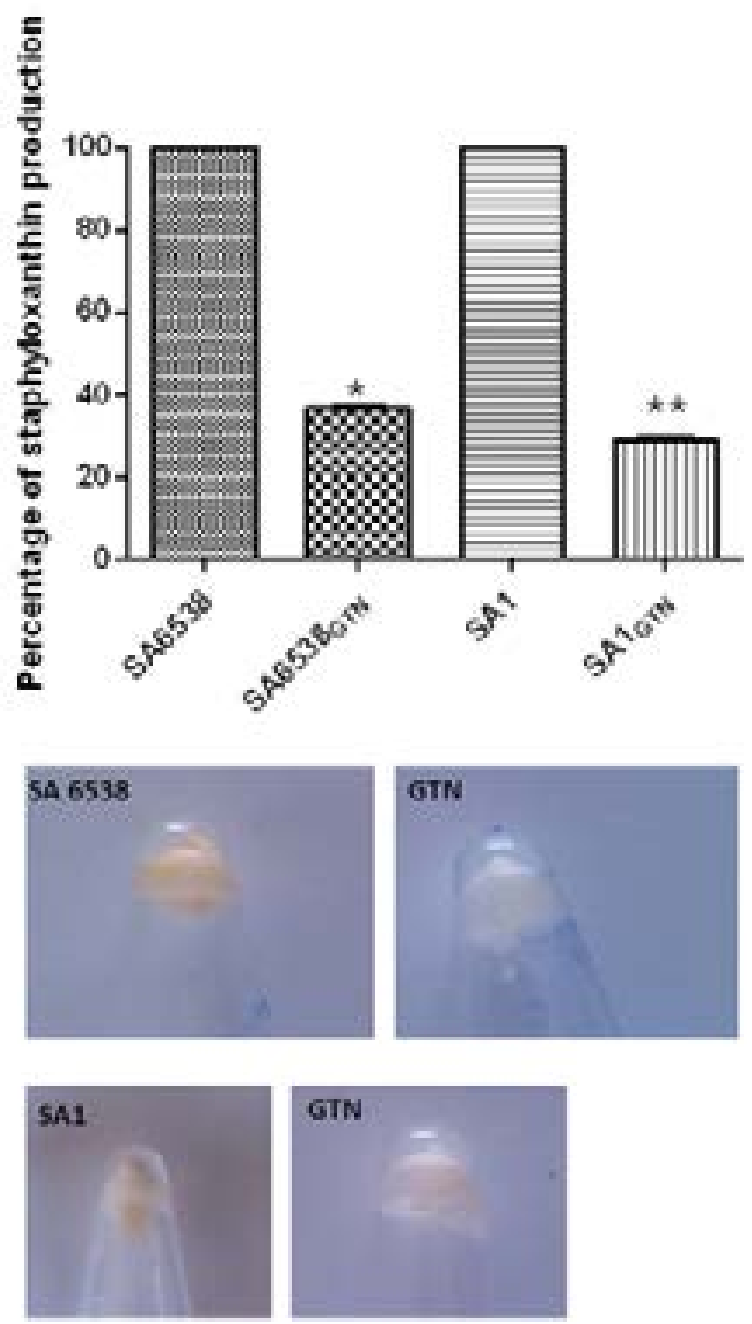

Figure 2. Inhibition of Staphyloxanthin production by $1 / 4$ MIC of GTN. ${ }^{*}$, significant $P<0.05$.

GTN decreases the tolerance against oxidative stress The inhibitory activity of GTN against the tolerance to oxidative stress was investigated by testing the augmen- tation of hydrogen peroxide on the growth of $S$. aureus by GTN. GTN showed a significant decrease in the tolerance to oxidative stress by $36.94 \%$ in $S$. aureus ATCC 6538 and $42.6 \%$ in the clinical isolate SA1 (Fig. 3). 


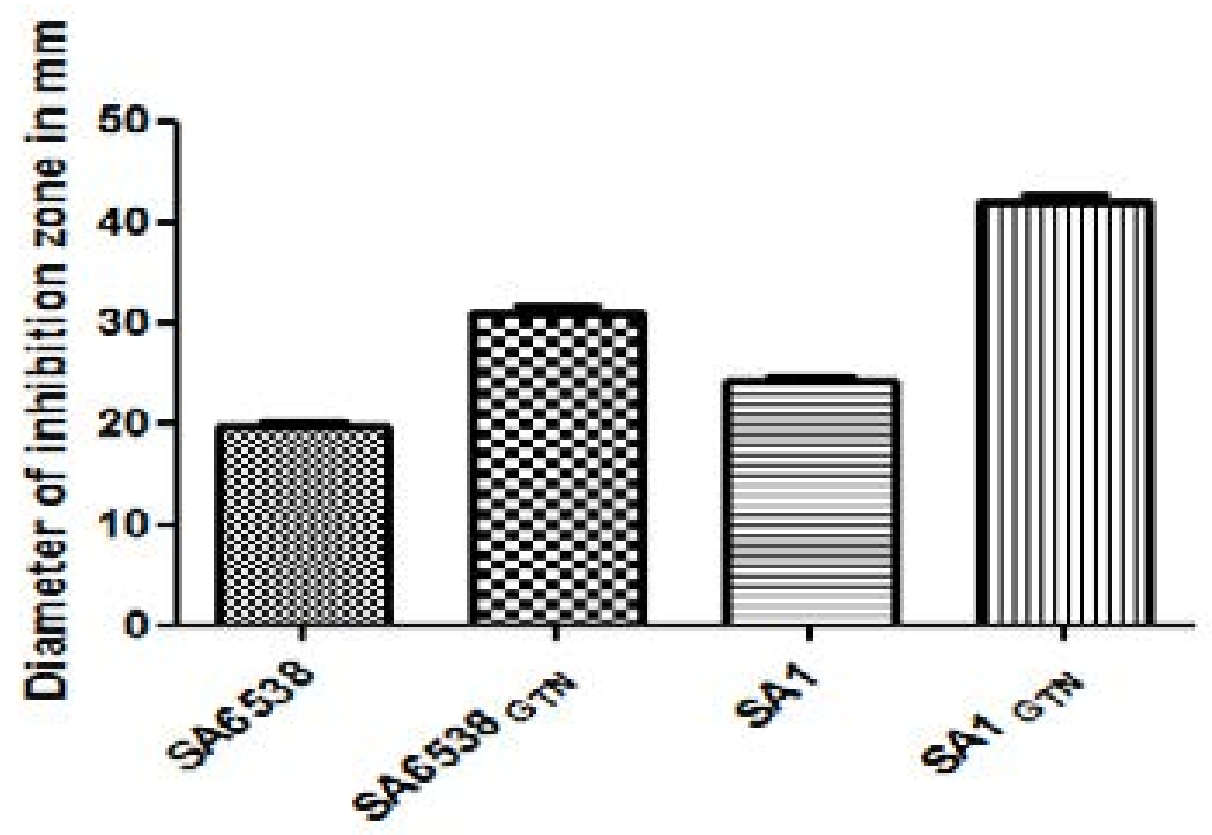

Figure 3. Reduction of tolerance to oxidative stress by $1 / 4$ MIC of GTN. *, significant $P<0.05$.

\section{Docking study}

The binding mode of GTN was studied using molecular docking study. GTN has nine hydrogen bonds with Arg45, Tyr129, Gln165, Asn168, Val 133 and Tyr248 elec- trostatic interaction with Arg45 and Asp48 and pi-cation interaction of the nitrogen atom with Tyr183 resulting in docking score of -99.658 (Fig. 4). GTN can effectively interact with the dehydrosqualene synthase active site.
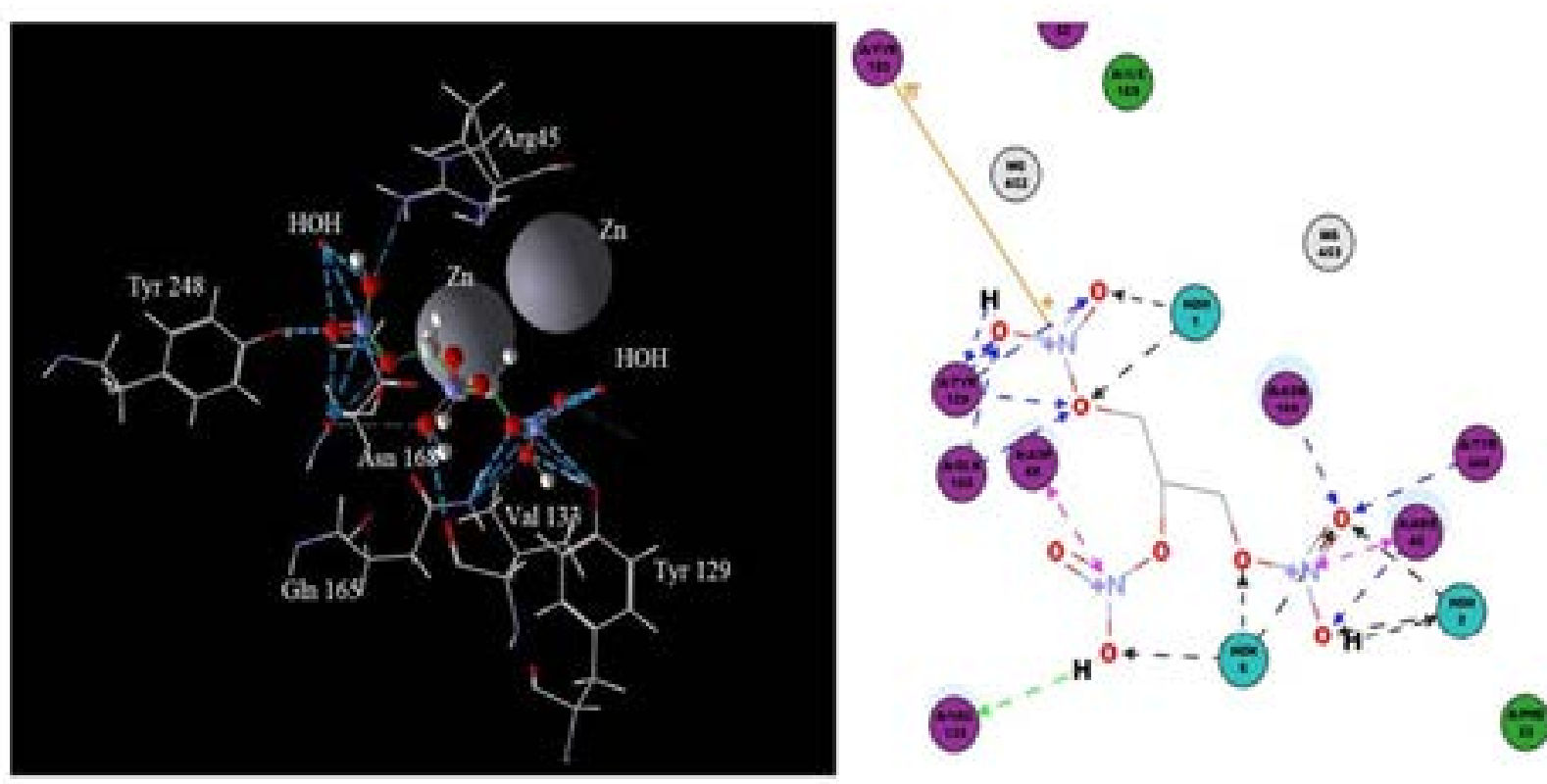

Figure 4. The Molecular docking of GTN into the active site of dehydrosqualene synthase 3D (Left) and 2D schematic view of the binding (Right). 


\section{Discussion}

The rate of methicillin resistance among $S$. aureus reached a peak in such a way that the mortality rate in the U.S. due to invasive MRSA exceeds that due to $\operatorname{AIDS}^{27,28}$. This problem needs urgent action for development of other therapeutic approaches ${ }^{29}$. One of these approaches is the search for anti-virulence drugs. In our study, GTN could inhibit the growth of $S$. aureus 6538 and the clinical isolate SA1 at $0.5 \mathrm{mg} / \mathrm{ml}$. The anti-virulence potential of GTN was investigated at $0.125 \mathrm{mg} / \mathrm{ml}$. The capability of $S$. aureus to produce virulence factors is linked to its pathogenicity. These factors include biofilm formation and the antioxidant pigment staphyloxanthin ${ }^{30}$.

Staphyloxanthin protects $S$. aureus from phagocytosis. It was reported that the strains of $S$. aureus that are deficient in staphyloxanthin production are sensitive to killing by reactive oxygen species produced by neutrophils and are defective in their ability to form skin abscesses ${ }^{1}$.

GTN exerted biofilm inhibiting activity that ranged between $52.64 \%$ and $69.16 \%$. The anti-biofilm activity of GTN against $S$. aureus was previously reported as a catheter lock solution in combination with ethanol and citrate $^{31}$. The anti-biofilm activity of GTN was further explored by microscopic examination of biofilms formed on glass cover slips treated and untreated with GTN. The treated sample showed very few scattered cells in contrast to the dense compact cells in the control sample.

In this study, the biological activity of GTN on the production of staphyloxanthin pigment was inspected by extraction of the pigment from GTN treated and untreated cells. The pigment in GTN treated cells was reduced to the range of $63.37 \%$ to $70.98 \%$. Furthermore, the in vitro susceptibility to oxidants was tested by investigation of the augmentation of the sensitivity to hydrogen peroxide. The susceptibility to hydrogen peroxide was increased between $36.94 \%$ and $42.6 \%$. This may be due to blockade of staphyloxanthin pigment. This was in accordance with the finding of Liu et al. ${ }^{32}$

Dehydrosqualene synthase is an enzyme that is involved in the first step of staphyloxanthin biosynthesis ${ }^{13}$. As a result, the interaction of GTN with dehydrosqualene synthase enzyme was investigated. Docking study was made to identify the ability of GTN to bind to the active site of dehydrosqualene synthase. GTN was found to attach to the enzyme by a combination of hydrogen bonds, electrostatic interaction and pi-cation interaction. In other terms, GTN may be a competitive inhibitor of this enzyme leading to inhibition of staphyloxanthin synthesis.

Interestingly, the anti-virulence activity of GTN was exerted at $0.125 \mathrm{mg} / \mathrm{ml}$, a much lower concentration of GTN that is approved by FDA for topical use (up to $0.4 \%$ or $4 \mathrm{mg} / \mathrm{ml}$ ).

Repurposing GTN as an anti-virulence agent is advantageous. It is an FDA approved whose pharmacological and toxicological properties are well-known. Moreover, using FDA approved drug saves the high costs and shortens the long development times to produce new antimicrobials or virulence inhibitors ${ }^{33}$. Our previous studies showed that GTN is a potent inhibitor of quorum sensing and virulence in Pseudomonas aeruginosa and Serratia marcescens $s^{34,35}$. The rationale of repurposing drugs for use as anti-virulence agents against Stapylococcus aureus was previously reported. Thus, diflunisal; the non-steroidal anti-inflammatory drug was reported as a potent inhibitor of virulence in USA300 strain of methicillin-resistant S. aureus $^{36}$. However, further clinical trials are necessary to test the clinical efficacy of GTN in laboratory animals and human volunteers.

\section{Conclusion}

GTN is a potential anti-virulence agent that can be useful in the treatment of $S$. aureus topical infections.

\section{Acknowledgement}

To the Department of Microbiology, Faculty of Pharmacy, Mansoura University for providing us with the standard strain Staphylococcus aureus ATCC 6538.

\section{Conflict of interest}

The authors declare that they have no conflict of interest.

\section{Funding source}

There was no funding for this work.

\section{References}

1. Liu CI, Liu GY, Song Y, Yin F, Hensler ME, Jeng WY et al. A cholesterol biosynthesis inhibitor blocks Staphylococcus aureus virulence. Science. 2008; 319:1391-1394. doi: 10.1126/science. 1153018 .

2. Levy SB, Marshall B. Antibacterial resistance world- 
wide: causes,challenges and responses. Nature Medicine. 2004; 10:S122-S129. doi:10.1038/nm1145

3. Cegelski L, Marshall GR, Eldridge GR, Hultgren SJ. The biology and future prospects of antivirulence therapies. Nature Reviews Microbiology. 2008; 6:17-27. doi: 10.1038/nrmicro1818

4. Hentzer M, Riedel K, Rasmussen TB, Heydorn A, Andersen JB, Parsek MR et al. Inhibition of quorum sensing in Pseudomonas aeruginosa biofilm bacteria by a halogenated furanone compound. Microbiology. 2002; 148:87-102. doi:10.1099/00221287-148-1-87

5. Daum RS. Removing the golden coat of Staphylococcus aureus. The New England Journal of Medicine. 2008; 359: 85-87. doi: 10.1056/NEJMcibr0803278.

6. Pacheco RL, Lobo RD, Oliveira MS, Farina EF, Santos CR, Costa SF et al. Methicillin-resistant Staphylococcus aureus (MRSA) carriage in a dermatology unit. Clinics (Sao Paulo). 2011; 66: 2071-2077. doi: 10.1590/S180759322011001200012

7. Karamatsu ML, Thorp AW, Brown L. Changes in community-associated methicillin-resistant Staphylococcus aureus skin and soft tissue infections presenting to the pediatric emergency department: comparing 2003 to 2008. Pediatrics Emergency Care. 2012; 28: 131-135. doi: 10.1097/ PEC.0b013e318243fa36.

8. Okesola A O. Community-acquired methicillin-resistant Staphylococcus aureus - a review of literature. African Journal of Medicine and Medical Sciences. 2011; 40: 97-107.

9. Dhand A, Sakoulas G. Reduced vancomycin susceptibility among clinical Staphylococcus aureus isolates ('the MIC Creep'): implications for therapy. F1000 Medicine Reports. 2012; 4: 4. doi: 10.3410/M4-4.

10. Stefani S, Chung DR, Lindsay JA, Friedrich AW, Kearns AM, Westh $\mathrm{H}$ et al. Meticillin-resistant Staphylococcus aureus (MRSA): global epidemiology and harmonisation of typing methods. International Journal of Antimicrobial Agents. 2012; 39: 273-282. doi: 10.1016/j.ijantimicag.2011.09.030.

11. Nuno G, Zampini IC, Ordonez RM, Alberto MR, Arias ME, Isla MI. Antioxidant/antibacterial activities of a topical phytopharmaceutical formulation containing a standardized extract of Baccharis incarum, an extremophile plant species from Argentine Puna. Phytotherapy Researh. 2012; 26: 1759-1767. doi: 10.1002/ptr.4633.

12. Liu GY, Essex A, Buchanan JT, Datta V, Hoffman HM, Bastian JF et al. Staphylococcus aureus golden pigment impairs neutrophil killing and promotes virulence through its anti-oxidant activity. Journal of Experimental Medicine. 2005; 202: 209-215. doi:10.1084/jem.20050846

13. Clauditz A, Resch A, Wieland KP, Peschel A, Götz F. Staphyloxanthin plays a role in the fitness of Staphylococcus aureus and its ability to cope with oxidative stress. Infection and Immunity. 2006; 74:4950-4953. doi: 10.1128/ IAI.00204-06

14. Song Y, Liu CI, Lin FY, No JH, Hensler M, Liu YL et al. Inhibition of staphyloxanthin virulence factor biosynthesis in Staphylococcus aureus: in vitro, in vivo, and crystallographic results. Journal of Medicinal Chemistry. 2009; 52:3869-3880. doi: 10.1021/jm9001764.

15. Otto M. Staphylococcal biofilms. Current Topics in Microbiology and Immunology 2008, 322: 207-228.

16. Sakai K, Koyama N, Fukuda T, Mori Y, Onaka H, Tomoda H. Search method for inhibitors of staphyloxanthin production by methicillin-resistant Staphylococcus aureus. Biologial \& Pharmaceutial Bulletin, 2012; 35:48-53

17. Willmot M, Ghadami A, Whysall B, Clarke W, Wardlaw J, Bath PM. Transdermal glyceryl trinitrate lowers blood pressure and maintains cerebral blood flow in recent stroke. Hypertension, 2006; 47(6):1209-1215. doi: 10.1161/01.HYP.0000223024.02939.1e

18. Rosenblatt J, Reitzel RA, Raad I. Caprylic acid and glyceryl trinitrate combination for eradication of biofilm. Antimicrobial Agents and Chemotherapy. 2015; 59:1786-1788. 19. Fenton C, Wellington K, Easthope SE. 0.4\% nitroglycerin ointment: in the treatment of chronic anal fissure pain. Drugs, 2006; 66:343-349. doi: 10.1128/AAC.0456114.

20. Clinical and Laboratory Standards Institute. Methods for dilution anti-microbial susceptibility tests for bacteria that grow aerobically; Approvated standard, CLSI Document M07-A9, Vol. 2012; 32, No. 3. Wayne, PA,USA.

21. Clinical and Laboratory Standards Institute. Performance standards for antimicrobial susceptibility testing, $17^{\text {th }}$ informational supplement. CLSI Document M100-S23. 2013; Wayne, USA

22. Stepanovic S, Vukovic D, Hola V, Di Bonaventura G, Djukić S, Cirković I, Ruzicka F. Quantification of biofilm in microtiter plates: overview of testing conditions and practical recommendations for assessment of biofilm production by Staphylococci. Acta Pathologica, Microbiologica, et Immunologica Scandinavica, 2007; 115(8): 891-899. doi:10.1111/j.1600-0463.2007. apm 630.x

23. Hassett DJ, Schweizer HP, Ohman DE. Pseudomonas aeruginosa sod $\mathrm{A}$ and $\operatorname{sodB}$ mutants defective in manga- 
nese- and iron-cofactored superoxide dismutase activity demonstrate the importance of the iron-cofactored form in aerobic metabolism. Journal of Bacteriology. 1995; 177: 6330-6337.

24. Chia IL, George YL, Yongcheng S, Fenglin Y, Mary EH, Wen YJ et al. A Cholesterol Biosynthesis Inhibitor Blocks Staphylococcus aureus Virulence. Science. 2008; 319(5868): 1391-1394. doi: 10.1126/science.1153018.

25. Marvin 17.14.0, 2016, ChemAxon: http://www.chemaxon.com

26. Thomsen R, Christensen MH. MolDock: A New Technique for High-Accuracy Molecular Docking. Journal of Medicinal Chemistry. 2006; 49: 3315-3321. doi 10.1021/ jm051197e

27. Bancroft EA. Anti-microbial resistance: It's not just for hospitals. Journal of the American Medical Association. 2007; 298:1803- 1804. doi:10.1001/jama.298.15.1803

28. Klevens RM, Morrison MA, Nadle J, Petit S, Gershman K, Ray $S$ et al. Invasive methicillin resistant Staphylococcus aureus infections in the United States. Journal of the American Medical Association. 2007; 298:1763-1771. doi:10.1001/jama.298.15.1763

29. WHO. Global priority list of antibiotic-resistant bacteria to guide research, discovery, and development of new antibiotics. 2017; 1-7.

30. Lowy FD. Staphylococcus aureus infections. The New England Journal of Medicine, 1998; 339: 520-532. doi:10.1056/ NEJM199808203390806
31. Rosenblatt J, Reitzel R, Dvorak T, Jiang Y, Hachem RY, Raad II. Glyceryl trinitrate complements citrate and ethanol in a novel anti-microbial catheter lock solution to eradicate biofilm organisms. Antimicrobial Agents and Chemotherapy. 2013; 57(8):3555-60. doi: 10.1128/AAC.0022913.

32. Liu GY, Essex A, Buchanan JT, Datta V, Hoffman HM, Bastian JF. Staphylococcus aureus golden pigment impairs neutrophil killing and promotes virulence through its antioxidant activity. Journal of Experimental Medicine. 2005; 202: 209-215. doi: 10.1084/jem.20050846

33. Mullard A. Drug repurposing programmes get lift off. Nature Reviews Drug Discovery. 2012; 11: 505-506. doi: $10.1038 / \operatorname{nrd} 3776$.

34. Abbas HA, Shaldam MA. Glyceryl trinitrate is a novel inhibitor of quorum sensing in Pseudomonas aeruginosa. African Health Sciences. 2016; 16(4): 1109-1117. doi: 10.4314/ ahs.v16i4.29

35. Abbas HA, Elsherbini AM. Silencing the noscomial pathogen Serratia marscescens by glyceryl trinitrate. African Health Sciences, 2018; 18(1):1-10. doi: 10.4314/ahs.v18i1.2 36. Khodaverdian V, Pesho M, Truitt B, Bollinger L, Patel P, Nithianantham S, et al. Discovery of antivirulence agents against methicillin-resistant Staphylococcus aureus. Antimicrobial Agents and Chemotherapy, 2013; 57 3645-3652 doi: 10.1128/AAC.00269-13 